\title{
Hybrid Plasmonic Waveguide Devices for Silicon on Insulator Platform
}

\author{
M. Z. Alam, J. S. Aitchison and M. Mojahedi \\ Department of Electrical and Computer Engineering, University of Toronto, Toronto, Ontario, Canada, M5S $3 G 4$ \\ malam@waves.utoronto.ca
}

\begin{abstract}
Properties of the modes supported by the hybrid metal-low-high index waveguides are strongly polarization dependent. We present designs of a number of hybrid waveguide devices for silicon on insulator platform that utilize this property.

OCIS codes: (240.6680) Optics at surfaces: Surface plasmons; (310.6628) Subwavelength structures, nanostructures; (130.3120) Integrated optics.
\end{abstract}

\section{Introduction}

Silicon on insulator (SOI) has emerged as a very attractive platform for integrated optics. SOI devices are compact, suffer very low loss, and offer the possibility of integration of photonics and electronics. Another promising photonic technology is plasmonics i.e. applications of surface plasmon (SP) at optical frequencies. Plasmonics can offer sub-diffraction confinement which is not possible by conventional optics. Because of the high field confinement and presence of a metal surface, SP is also very suitable for biosensing and many other applications where extreme field confinement is critical. The most serious limitation of plasmonics is the large propagation loss of plasmonic waveguides. At optical wavelength metal permittivity is complex with a large imaginary part. As a result SP suffers from significant propagation loss. Recently, we proposed a waveguide that supports a hybrid mode resulting from the coupling of a dielectric waveguide mode with a SP mode $[1,2]$. The guide offers a better compromise between loss and confinement than existing plasmonic guides [3]. Since our early work on hybrid guides, significant amount of work on the hybrid guide has been reported and a number of different types of hybrid guide have been proposed [3-4]. One recently proposed hybrid guide that is very suitable for integration with SOI technology [4] is shown in Fig. 1(a). It consists of a silicon layer of dimension $w \times d$ separated from a silver film of dimension $w \times t$ by a silica spacer of dimension $w \times h$.
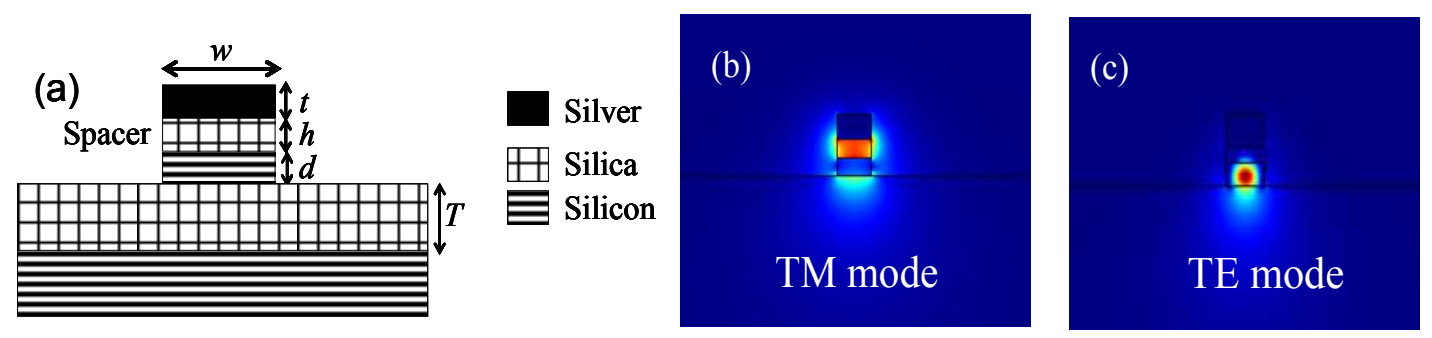

Fig. 1 (a) Schematic of the hybrid waveguide. (b) Electric field intensity for the TM mode. (c) Electric field intensity for the TE mode. Waveguide dimensions are $\mathrm{w}=350 \mathrm{~nm}, \mathrm{t}=200 \mathrm{~nm}, \mathrm{~h}=150 \mathrm{~nm}, \mathrm{~d}=150 \mathrm{~nm}$, and $\mathrm{T}=2 \mu \mathrm{m}$. Wavelength of operation is $1550 \mathrm{~nm}$.

Figures 1(b) and 1(c) show the electric field intensity for the TM and TE modes supported by the structure, respectively. The TE mode is similar to that of a dielectric waveguide mode and is confined in the high index region (silicon). In contrast, the TM mode is confined in the low index spacer layer between silicon and silver. Since the two modes are confined in two different regions, changes of waveguide dimensions affect the two modes differently. This strong polarization dependency of the hybrid guide has not received any attention so far.

To illustrate the usefulness of this polarization dependency, here we present a number of hybrid integrated optical devices that exploit this property. The devices we propose are TE-pass polarizer, TM-pass polarizer, and polarization independent directional coupler. All these devices are intended for applications on an SOI platform and as such are compatible with existing mature electronic technology. The designs and analyses are carried out with the help of finite difference time domain (FDTD) code Lumerical. Properties of silicon and silica are taken from [5] and the properties of silver are taken from [6]. A non-uniform mesh with mesh size of $2 \mathrm{~nm}$ around the metal is used in all these simulations. The simulations are performed on the high performance computing facility WestGrid. 


\section{ITuB4.pdf}

\section{TM-Pass Polarizer}

When the spacer thickness $(h)$ for the hybrid guide shown in Fig. 1(a) is small, the TM mode is highly confined in the spacer layer, while the TE mode is pushed into the substrate and approaches the cutoff. Therefore, the hybrid waveguide acts as a TM-pass polarizer for a small spacer thickness. Figure 2(a) shows the schematic of a complete hybrid TM-pass polarizer. It consists of a hybrid waveguide [shown in Fig. 2(b)] inserted between two input/output silicon waveguides [shown in Fig. 2(c)]. Since the dimensions and mode sizes of the hybrid guide are comparable to those of the silicon waveguide, no taper is used between the silicon waveguides and the hybrid section.
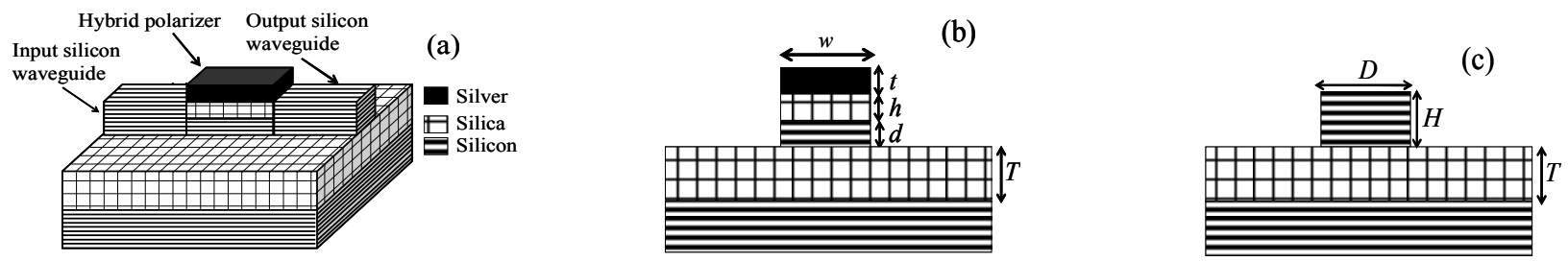

Fig. 2. (a) Schematic of the complete TM-pass polarizer. (b) Cross section of the hybrid waveguide. (c) Cross section of the input and output silicon waveguides.

Figure 3 shows the insertion loss of the TM and TE modes for an $18 \mu \mathrm{m}$ long TM-pass polarizer. Performance of our proposed hybrid TM-pass polarizer compares favorably with previously reported TM-pass polarizers [7], especially for small buried oxide thickness e.g., $T=1 \mu \mathrm{m}$.
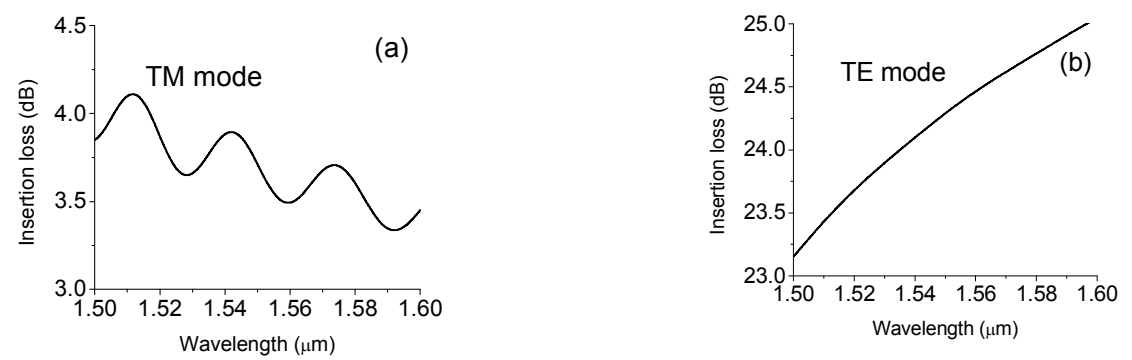

Fig. 3. Insertion losses of the TM-pass polarizer for (a) TM mode (b) TE mode. Device dimensions are $w=350 \mathrm{~nm}, t=200 \mathrm{~nm}, h=100$ $\mathrm{nm}, d=100 \mathrm{~nm}$, and $T=1 \mu \mathrm{m}$. Input and output waveguide dimensions are $D=350 \mathrm{~nm}$ and $H=300 \mathrm{~nm}$.

\section{TE-Pass Polarizer}

By choosing the hybrid waveguide dimensions properly, it is possible to make the loss of the TM mode much larger than that of the TE mode. The device shown in Fig. 2 then acts as a TE-pass polarizer. Figures 4(a) and 4(b) show the insertion loss for the TE and TM modes respectively for a $25 \mu \mathrm{m}$ long polarizer. Chromium is chosen instead of silver for this design to minimize the device length.
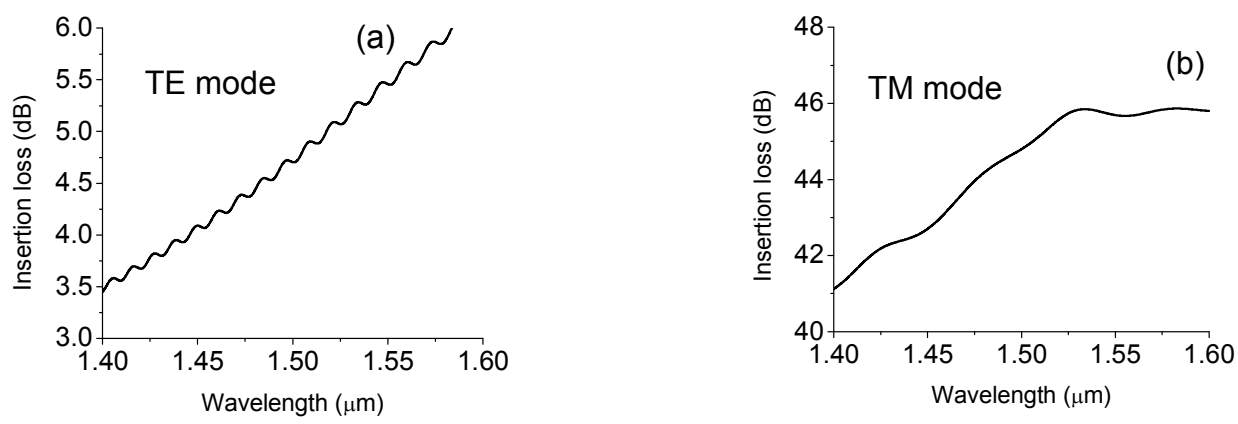

Fig. 4. Insertion losses of the (a) TE and (b) TM modes for our proposed TE-pass polarizer. Hybrid waveguide dimensions are $w=500$ $\mathrm{nm}, t=200 \mathrm{~nm}, h=300 \mathrm{~nm}, d=100 \mathrm{~nm}$, and $T=1 \mu \mathrm{m}$. Input and output waveguide dimensions are $D=350 \mathrm{~nm}$ and $H=300 \mathrm{~nm}$. 


\section{ITuB4.pdf}

As compared to our $25 \mu \mathrm{m}$ long proposed polarizer, the most compact broadband TE-pass polarizer on SOI reported to date has a length of $120 \mu \mathrm{m}$ [8]. Simulation results for that polarizer predicts an insertion loss of more than $30 \mathrm{~dB}$ for the TM mode and 2 to $4 \mathrm{~dB}$ for the TE mode over a bandwidth of $350 \mathrm{~nm}$. In contrast, as shown in Fig. 4, our proposed TE-pass polarizer has higher insertion loss for the TE mode (3.5 to $6 \mathrm{~dB})$, but also greater insertion loss for the TM mode (greater than $41 \mathrm{~dB}$ ) over more than $200 \mathrm{~nm}$ bandwidth; while at the same time our device length is approximately $20 \%$ of that reported in [8].

\section{Polarization Independent Directional Coupler}

The high dielectric contrast of silicon and silica makes SOI devices highly polarization dependent. Moreover, polarization state of a standard optical fiber may vary randomly and connecting such a fiber directly to an SOI optical chip may disrupt the proper operation of the latter. One possible solution to this problem is to separate the two polarizations at the input of SOI chip and process them separately. This results in an increased system size and complexity. Another solution is to make the SOI devices polarization independent. Although zero birefringence can be achieved for SOI waveguides in a relatively straight forward manner [9], implementing a polarization independent SOI directional coupler is a challenging task. A hybrid plasmonic waveguide directional coupler such as the one shown in Fig. 5 (a) can be used to achieve polarization independent operation. The device consists of two hybrid waveguides separated by distance $S$. Figures 5(b) and 5(c) show the effects of variations of silica and silicon thicknesses $(h$ and $d$ ) on coupling lengths $(L)$ for the TE and TM modes. Here, the coupling length $(L)$ is defined as the length required for complete power transfer from one guide to the other. For $h=45 \mathrm{~nm}$ and $d=340 \mathrm{~nm}$ the coupling lengths for both modes are equal and the device becomes polarization independent at $1.55 \mu \mathrm{m}$. Coupling length in this case is approximately $10.8 \mu \mathrm{m}$.
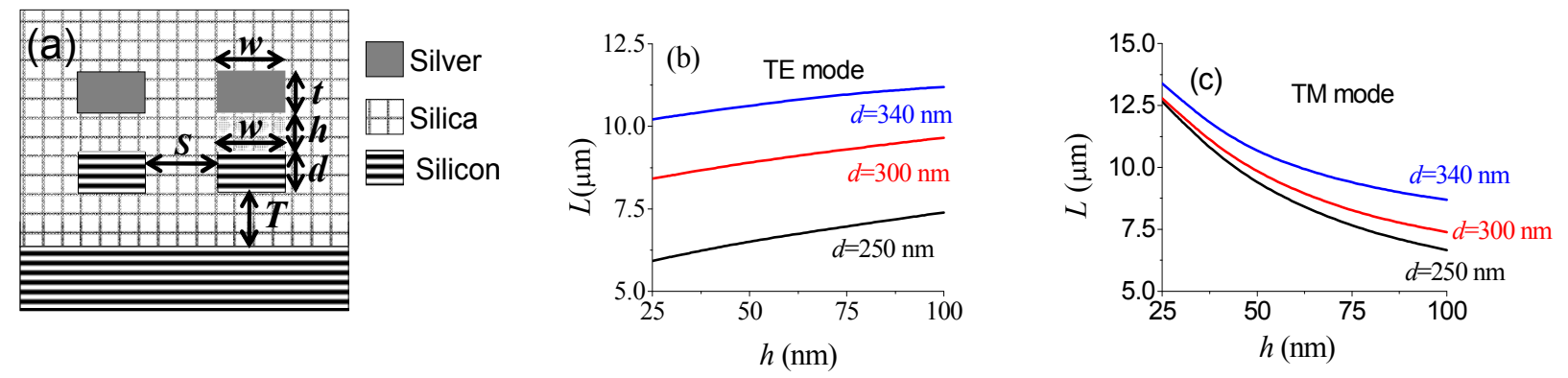

Fig.5. (a) Cross section of the hybrid directional coupler. (b) and (c) Variations of coupling length $(L)$ with varying $h$ and $d$ for the TE mode and the TM mode. Other waveguide dimensions are $w=340 \mathrm{~nm}, t=200 \mathrm{~nm}, S=200 \mathrm{~nm}, T=1 \mu \mathrm{m}$. Wavelength of operation is $1.55 \mu \mathrm{m}$.

\section{Conclusion}

We have presented designs of a number of devices which take advantage of hybrid plasmonic waveguides. These devices utilize the strong polarization dependency of the hybrid plasmonic waveguides. The devices are very compact and are fully compatible with SOI fabrication technology. They can provide improved performance and new functionalities. Many other devices, for example polarization rotator and highly sensitive biosensor can also be implemented using the polarization dependency of the hybrid waveguide.

\section{References}

[1] M Z. Alam, J. Meier, J S. Aitchison and M Mojahedi, “Super mode propagation in low index medium," CLEO/QELS (2007).

[2] M. Z. Alam, J. Meier, J. S. Aitchison and M. Mojahedi, "Propagation characteristics of hybrid modes supported by metal-low-high index waveguides and bends," Opt. Expr. 18, 12971-12979 (2010).

[3] R. F. Oulton, V. J. Sorger, D. A. Genov, D. F. P. Pile, X. Zhang, “A hybrid plasmonic waveguide for subwavelength confinement and long range propagation," Nat. Photon. 2, 496-500 (2008).

[4] D. Dai, and S. He, "A silicon-based hybrid plasmonic waveguide with a metal cap for nano-scale light confinement," Opt. Exp. 17, 1664616653 (2008).

[5] E. D. Palik, Handbook of optical constants of solids, Academic Press Inc., (1985).

[6] P. B. Johnson, R. W. Christy, "Optical constants of noble metals," Phys. Rev. B 6, 4370-4379 (1972).

[7] Q. Wang, and S-T. Ho, "Ultra compact TM-pass silicon nanophotonic waveguide polarizer and design," IEEE J. Photon. 2, 49-56 (2010).

[8] I. Avrutsky, "Integrated optical polarizer for silicon-on-insulator waveguides using evanescent wave coupling to gap plasmon-polaritons," IEEE J. of Quantum Electron. 14, 1509-1514, (2008).

[9]S. T. Lim, C. E. Png, E. A. Ong, "Single mode polarization independent submicron silicon waveguide based on geometrical adjustments," Opt. Exp. 15, 11061-11072 (2007). 